\title{
Dolor y discapacidad cervical de los trabajadores públicos usuarios de pantallas de visualización de datos
}

\author{
Pain and cervical disability among public workers who use \\ data visualization display terminals
}

Tania García-Remeseiro (https://orcid.org/0000-0002-0513-2227) ${ }^{1}$

Águeda Gutiérrez-Sánchez (https://orcid.org/0000-0003-2414-2882) ${ }^{1}$

Rui Garganta (https://orcid.org/0000-0001-5178-5387) ${ }^{2}$

Diego Alonso-Fernández (https://orcid.org/0000-0003-4992-8080) ${ }^{1}$

${ }^{1}$ Universidade de Vigo Campus Universitario A Xunqueira 36005. Pontevedra Espanha. tgarcia@uvigo.es

${ }^{2}$ Universidade do Porto. Porto Portugal.

\begin{abstract}
Work-related neck/shoulder disorders are considered an important health issue. This study is aimed at establishing the pain and cervical disability patterns of workers with visual display terminals, as well as at determining the factors that mostly affect the onset of a new episode of back pain. A descriptive, correlational study was carried out on a sample of 88 workers who use visual display terminals, of the Ministry of Health (Xunta de Galicia). The workers completed the following questionnaires: "Neck Disability Index Scale," "Visual Analog Scale," "12-item Short Form Health Survey," and an individual postural analysis was conducted. For the comparative anal$y$ sis, the Student's t-test, and the Mann-Whitney $U$ test were performed. A binary logistic regression analysis was used to extract a predictive model of a cervical pain episode, and $58 \%$ reported cervical pain. There were no differences between men and women. The variables that best predict the onset of a new episode of pain are the level of disability, and the quality of physical life. Study results indicate that a worker who uses visual display terminals, with high levels of cervical disability and low values of physical quality of life, is more likely to suffer an episode of back pain.
\end{abstract}

Key words Musculoskeletal pain, Posture, Neck pain, Photogrammetry, Work
Resumen Los trastornos del cuello-hombro relacionados con el trabajo son un importante problema de salud. El objetivo de este estudio es establecer el patrón de dolor y discapacidad cervical de trabajadores con pantallas de visualización de datos, así como determinar los factores que predominan en la aparición de un nuevo episodio de dolor de espalda. Se realizó un estudio descriptivo-correlacional, en una muestra de 88 trabajadores usuarios de ordenadores de la Consellería de Sanidade (Xunta de Galicia). Los trabajadores respondieron los cuestionarios: "Neck Disability Index", "Escala analógica visual", "12-item Short Form Health Survey" y se realizó un análisis postural. Para el análisis comparativo se realizaron las pruebas T Student y U de Mann-Whitney. Un análisis de regresión logística binaria se utilizó para la extracción de un modelo predictivo de episodio de dolor cervical. El 58\% refirieron dolor cervical. No existieron diferencias significativas entre hombres y mujeres. Las variables que mejor predicen la aparición de un nuevo episodio de dolor son el nivel de discapacidad y la calidad de vida física. Los resultados indican que un trabajador con altos niveles de discapacidad cervical y bajos valores de calidad de vida física, tiene mayor probabilidad de sufrir un episodio de dolor.

Palabras clave Dolor musculoesquelético, Postura, Dolor de cuello, Fotogrametría, Trabajo 


\section{Introducción}

Según el estudio Global Burden of Disease Study 2015, el dolor lumbar y el dolor de cuello fueron las principales causas de discapacidad en el mundo en $2015^{1}$. Existe una gran cantidad de literatura epidemiológica, que trata sobre los trastornos del cuello-hombro relacionados con el trabajo como un problema de salud importante en muchas ocupaciones ${ }^{2-5}$. Aun así, la relación con el trabajo en el sentido de una relación dosis-respuesta bien establecida o de una fracción etiológica calculada no está bien documentada. Esto se debe a varios factores que presentan diferentes grados de dificultad para su interpretación directa. Como es el caso del término "trastorno cuello-hombro", que abarca desde el dolor autoinformado hasta el diagnóstico clínico bien definido ${ }^{3}$.

En España, el padecer dolor de cuello va en aumento. Entre los años 2009 y 2012, el dolor de cuello como único cuadro clínico pasaba de un $7,86 \%$ a un $8,56 \%$. Y si estaba asociado a dolor en la zona lumbar pasaba de un $10,61 \%$ a un $11,12 \%{ }^{6}$. La prevalencia de todas las localizaciones del dolor aumenta con la edad, un nivel inferior de educación, la mala autopercepción de la salud, ser fumador, co-morbilidades, trastornos psicológicos (ansiedad o depresión), visitas al hospital y el fisioterapeuta y los fármacos consumidos para el dolor, en 2008/9, en comparación con $2011 / 12^{6}$.

En población trabajadora, diversos estudios han obtenido prevalencias de síntomas de las extremidades superiores de 20 a $30 \%$ o incluso más ${ }^{7}$. Además, dentro de ciertas ocupaciones la prevalencia es aún mayor que en la población general. Así, en un estudio de mujeres mayores de 45 años, usuarias de ordenador en los países de la Unión Europea, se encontró al 60\% de las mujeres con síntomas en el cuello.

Larsson et al. ${ }^{3}$ realizaron un trabajo de revisión en el que tratan de abordar la aparición de dolor de cuello-hombro, los factores de riesgo para el desarrollo de dolor de cuello-hombro y su relación con el trabajo. Identifican los siguientes factores de riesgo: sexo, movimientos repetitivos, demandas elevadas de fuerza, postura del trabajo, vibración, trabajo con el ordenador y factores psicosociales. En otro estudio de revisión realizado por McLean et al. ${ }^{9}$ se identificaron como factores de riesgo para el dolor inespecífico de cuello: el sexo femenino, la edad avanzada, las altas demandas laborales, el bajo apoyo social/laboral, ser ex-fumador, los antecedentes de trastornos de la espalda baja e historia previa de trastornos del cuello.

El objetivo de este estudio fue establecer el patrón de dolor y discapacidad cervical de los trabajadores públicos que trabajan con pantallas de visualización de datos (PVD), así como determinar los factores que predominan en la aparición de un nuevo episodio de dolor de espalda en dichos trabajadores en función del sexo.

\section{Material y métodos}

\section{Participantes}

La muestra estuvo constituida por 88 trabajadores de PVD de la Consellería de Sanidade (Xunta de Galicia, España). Siendo 21 hombres y 67 mujeres, con edades comprendidas entre los 27 y los 65 años, siendo la media de edad de 48,31 años.

El cálculo de la muestra se hizo de forma global para hombres y mujeres conjuntamente. De este modo los porcentajes de hombres y mujeres en la muestra ( $24 \%$ y $76 \%$ respectivamente), estiman los porcentajes de la población de referencia que cuenta con un total de 405 trabajadores, 316 mujeres $(78 \%)$ y 89 hombres (22\%).

La estimación del tamaño de la muestra se realizó mediante la siguiente fórmula:

$$
\mathrm{n}=\frac{N \sigma^{2} Z^{2}}{e^{2}(N-1)+\sigma^{2} Z^{2}}
$$

Donde: $\mathrm{n}=\mathrm{el}$ tamaño de la muestra, $\mathrm{N}=$ tamaño de la población, $\sigma=$ desviación estándar de la población, $\mathrm{Z}=$ Valor obtenido mediante niveles de confianza, e=Límite aceptable de error muestral. Por lo tanto, calculamos el tamaño de la muestra de una población de 405 elementos con un nivel de confianza del $95 \%$ y un porcentaje de error del $9,25 \%$, obteniendo una $\mathrm{n}=88$.

Los criterios de exclusión se vincularon a haber sufrido patologías que afectan a la postura corporal y que por tanto podrían convertirse en un sesgo para los resultados: antecedentes de fractura cervical o trauma, cirugía cervical, escoliosis idiopática, cáncer de hueso, tortícolis espasmódica, trastorno neurológico del movimiento, enfermedad del sistema nervioso central, dificultades respiratorias persistentes durante los últimos cinco años, deficiencia auditiva que requiera el uso de audífono o cirugía temporomandibular. 


\section{Escala visual analógica (EVA)}

La intensidad del dolor se midió a través de la escala EVA ${ }^{10}$. Permite obtener una puntuación objetiva de un síntoma tan subjetivo e individual como es el dolor. Su principal ventaja estriba en el hecho de que no requiere habilidades verbales o de lectura y es suficientemente versátil para ser empleada en diferentes situaciones ${ }^{11}$. Es una de las escalas más frecuentemente utilizadas debido a su validez y fiabilidad ${ }^{12,13}$.

\section{Escala Neck Disability Index (NDI)}

El índice de discapacidad cervical (NDI) es el primer instrumento diseñado para evaluar la autopercepción de la discapacidad en los pacientes con dolor de cuello ${ }^{14}$. El NDI es un instrumento relativamente corto, fácil de aplicar y que se aplica tanto en el ámbito de la investigación como en el ámbito clínico. Tiene fuertes propiedades psicométricas y ha demostrado ser muy sensible a los ensayos clínicos ${ }^{15}$. La interpretación del NDI se realiza de la siguiente manera: 0-4: sin discapacidad; 5-14: discapacidad leve; 15-24: discapacidad moderada; 25-34: discapacidad severa; más de 34: discapacidad completa. El estudio original informó de la fiabilidad test-retest de $0,89(\mathrm{p}<0,05)$. La consistencia interna se midió usando $\alpha$ de Cronbach, con un valor del índice total de $0,80^{14}$.

\section{2-item Short Form Health Survey (SF-12)}

El cuestionario de Calidad de Vida SF-12 ${ }^{16}$, corresponde a una versión práctica abreviada del original SF-36 ${ }^{17}$. Está formado por un subconjunto de 12 ítems del SF-36, a partir de los cuales se construyen los componentes sumarios físico (CSF) y mental (CSM) del SF-12 como únicas puntuaciones $^{18}$. Definiendo un estado positivo y negativo de la salud física y mental, por medio de ocho dimensiones (función física, rol físico, dolor corporal, salud mental, salud general, vitalidad, función social y rol emocional), algunos ítems del instrumento son: "En general, usted diría que su salud es", "Durante las 4 últimas semanas, ¿Hasta qué punto su salud física o los problemas emocionales han dificultado sus actividades sociales habituales con la familia, los amigos/as, los vecinos/as u otras personas?", "¿Tuvo dolor en alguna parte del cuerpo durante las 4 últimas semanas?". Las opciones de respuesta forman escalas de tipo Likert (donde el número de opciones varía de tres a seis puntos, dependiendo del ítem), que evalúan intensidad $y / o$ frecuencia del estado de salud de las personas ${ }^{19}$. Es uno de los instrumentos genéricos más utilizados en todo el mundo para la evaluación de la calidad de vida relacionada con la salud. La forma corta resultante logró R cuadrados múltiples de 0,911 y 0,918 en las predicciones de las puntuaciones del SF-36 componente físico y SF-36 componente mental, respectivamente. Las correlaciones test-retest (2 semanas) fueron de 0,89 y 0,76 para el componente físico y el componente mental respectivamente ${ }^{16}$.

\section{Software de evaluación postural (SAPO/PAS)}

La fotogrametría está siendo utilizada como herramienta de diagnóstico de los fisioterapeutas para medir la postura corporal ${ }^{20}$. El instrumento utilizado para realizar la evaluación postural de forma individualizada fue el software de evaluación postural (SAPO/PAS) ${ }^{21}$. Entre los métodos existentes, el software SAPO/PAS es una herramienta sencilla de manipulación, de acceso libre a través de Internet y desarrollado por investigadores de la Universidad de Sao Paulo (Brasil). Se basa fundamentalmente en la digitalización de imágenes y permite realizar varias funciones tales como la calibración de imágenes, el uso del zoom, la marcación libre de puntos, la medición de distancias y ángulos del cuerpo ${ }^{20}$.

Estas mediciones cuantitativas permiten a los fisioterapeutas, médicos e investigadores hacer una evaluación precisa de la postura y de cambios posturales que se producen tras un tratamiento y/o intervención.

\section{Procedimiento}

Este proyecto fue aprobado por el Comité Autonómico de Ética de Investigación de la Xunta de Galicia (España) y se realizó conforme a las directrices éticas para estudios de investigación recogidas en la declaración de Helsinki.

Todos los participantes fueron informados previamente de las características y objetivos del estudio aceptando su colaboración mediante la firma del correspondiente consentimiento informado. Posteriormente, respondieron a los cuestionarios EVA, NDI y SF-12 de manera individual. Se realizó un análisis postural a cada sujeto siguiendo el protocolo de Ferreira et al. ${ }^{21}$. Para marcar los puntos, se colocaron bolas de espuma de poliestireno (15 $\mathrm{mm}$ de circunferencia) con cinta adhesiva de doble cara. La cámara digital se colocó en el trípode (altura de 1,45 metros) con ángulos de 90 grados (la misma distancia). La cámara se colocó a tres metros del sujeto y se ajustó 
para ser perpendicular a los planos anatómicos de la materia. El zoom de la cámara se ajustó para permitir aproximadamente 0,5 metros de espacio libre debajo y por encima del sujeto para minimizar cualquier distorsión de las extremidades de imagen. Una línea vertical marcada con dos bolas de espuma de poliestireno se utilizó para la calibración vertical, colocada a una distancia de 0,5 metros.

La evaluadora era usuaria regular del software SAPO/PAS ${ }^{22}$. Las imágenes fueron calibradas según la distancia y la línea vertical. Se usó la función de zoom a $50 \%$. Se repitió el procedimiento y las pruebas fueron comparadas para evaluar la fiabilidad intraevaluador. La estimación de la fiabilidad se ha realizado a través del coeficiente de correlación intraclase (CCI), obteniendo un coeficiente de 0,941 , siendo considerado como excelente $^{23}$.

Se le realizó una fotografía a cada participante en bipedestación en el plano sagital y coronal, una vez marcados los puntos anatómicos de referencia. Los puntos de referencia fueron: tragus de la oreja, canto lateral del ojo, espinosa de C7, acromiones, glabela. Los participantes fueron fotografiados descalzos y en camiseta de tirantes para obtener una mejor observación de los puntos.

Se preservó en todo momento el anonimato de los participantes. Fueron identificados a través de un código durante las mediciones y en la base de datos electrónica. Toda su información fue asociada con ese código y no con sus datos personales.

Después de la realización de las fotografías se realizó la digitalización y análisis de los puntos mediante el software de evaluación postural (SAPO/PAS), generando una evaluación postural individual.

\section{Análisis estadístico}

Se analizaron como medida de tendencia central la media y como medidas de dispersión la desviación típica (DT). Los datos fueron sometidos a la prueba $Z$ de Kolmogorov-Smirnov para comprobar la normalidad. En el análisis comparativo se realizó la prueba $\mathrm{T}$ student para comparar medidas independientes y la $\mathrm{U}$ de Mann-Whitney para las variables que no mantenían una distribución normal u homogénea. Para detectar las variables predictivas de un mayor riesgo de episodio de dolor cervical, se ajustó un modelo de regresión logística binaria, mediante el método por pasos hacia delante-forward-. En los contrastes de hipótesis se consideró un nivel de significación estadística del 0,05 y los intervalos de confianza se calcularon al 95\%.

El análisis estadístico se realizó con el programa SPSS 21.0@ (Statistical Package for the Social Sciences) para Windows.

\section{Resultados}

La media de intensidad del dolor es de 2,73 para las mujeres y de 2,62 para los hombres (Tabla 1). El 28,4\% de los participantes están diagnosticados de alguna lesión o patología en la espalda y más de la mitad de los participantes (58\%), refirieron dolor de espalda en el último año que les dificulta las actividades de la vida diaria. En cuanto a la frecuencia en los episodios de dolor de espalda, el $45,5 \%$ refiere que al menos una vez tuvieron dolor en el último año, un 37,5\% varias veces durante el año, un 10,2\% frecuentemente y un $6,8 \%$ refiere padecer continuamente dolor. En relación a la duración del dolor, el 59,1\% de los participantes tienen un dolor que dura menos de 12 horas, el 9,1\% cuantifica la duración del dolor de 12-24 horas, el 19,3\% entre 1 y 7 días, el 6,8\% en más de una semana y tan solo el 5,7\% tiene un dolor con una duración de más de un mes. Además, cuando están inmersos en un episodio de dolor, el 69,3\% de los participantes utiliza fármacos para aliviar el dolor de espalda.

Al testar si hay diferencias en la intensidad del dolor entre los hombres y las mujeres, comprobamos que no hay diferencias estadísticamente significativas entre las medias de ambos grupos (Tabla 2).

En la escala NDI la media se sitúa en 4,26 con una desviación típica de 4,453, es decir, los participantes no tienen discapacidad cervical o bien tienen un grado de discapacidad leve. El mínimo estuvo en 0 (ninguna discapacidad) y el máximo en 21 (discapacidad moderada). Ninguno de los participantes en el estudio obtuvo grados de discapacidad severa o incapacidad completa.

En la Tabla 3, observamos que no existen diferencias estadísticamente significativas en relación al sexo en cuanto a la discapacidad cervical.

Existe una relación estadísticamente significativa entre el ángulo de la cabeza y la intensidad del dolor de los participantes (Tabla 4).

Con el fin de conocer las variables que explican mejor la probabilidad de sufrir un episodio de dolor $(0 / 1=$ No episodio dolor/Episodio de dolor) se realiza una regresión logística binaria. Se observa como las únicas variables que tienen un peso predictivo significativo fueron el compo- 
Tabla 1. Estadísticos descriptivos de la escala EVA y ángulos cervical, hombro y cabeza.

\begin{tabular}{lcccc}
\hline & Mínimo & Máximo & Media & DT \\
\hline EVA (mm) & 0 & 9 & 2,70 & 2,67 \\
Mujer & 0 & 9 & 2,73 & 2,72 \\
Hombre & 0 & 8 & 2,62 & 2,87 \\
Ángulo cervical(o) & 32,50 & 58,40 & 46,05 & 5,70 \\
$\quad$ Mujer & 32,50 & 58,40 & 45,97 & 5,80 \\
Hombre & 32,90 & 52,10 & 46,31 & 5,50 \\
Angulo hombro(o) & 21,40 & 88,40 & 54,19 & 14,36 \\
$\quad$ Mujer & 21,40 & 88,40 & 54,46 & 14,89 \\
Hombre & 21,40 & 79,70 & 53,37 & 12,82 \\
Angulo cabeza $\left(^{\circ}\right)$ & 2,90 & 43,60 & 23,70 & 8,09 \\
Mujer & 2,90 & 43,60 & 23,49 & 8,57 \\
Hombre & 11,30 & 38,80 & 24,40 & 6,43 \\
\hline
\end{tabular}

Fuente: Desarrollada por los autores.

Tabla 2. Prueba T: sexo y EVA.

\begin{tabular}{|c|c|c|c|c|c|c|}
\hline & \multirow[t]{2}{*}{ Sexo } & \multirow[t]{2}{*}{ Media } & \multicolumn{2}{|c|}{$\begin{array}{l}\text { Prueba de } \\
\text { Levene }\end{array}$} & \multicolumn{2}{|c|}{ Prueba T } \\
\hline & & & $\mathbf{F}$ & Sig & $t$ & Sig \\
\hline \multirow[t]{2}{*}{ EVA } & Hombre & 2,62 & 0,346 & 0,575 & $-0,167$ & 0,868 \\
\hline & Mujer & 2,73 & & & & \\
\hline
\end{tabular}

Tabla 4. Correlación entre ángulo y intensidad del dolor.

\begin{tabular}{|c|c|c|c|c|}
\hline & & $\begin{array}{c}\text { Angulo } \\
\text { cabeza }\end{array}$ & $\begin{array}{l}\text { Angulo } \\
\text { hombro }\end{array}$ & $\begin{array}{l}\text { Angulo } \\
\text { cervical }\end{array}$ \\
\hline \multirow[t]{2}{*}{ EVA } & $\begin{array}{l}\text { Coeficiente } \\
\text { correlación }\end{array}$ & $-0,291$ & 0,110 & $-0,111$ \\
\hline & Sig. & 0,006 & 0,307 & 0,303 \\
\hline
\end{tabular}

nente sumario físico de la calidad de vida (CSF) y la discapacidad cervical. El modelo clasificó correctamente al 68,2 \% de los casos. La prue- ba de Hosmer y Lemeshow indicó la bondad de ajuste del modelo (Sig=0,489). El R cuadrado de Nagelkerke indicó que el modelo explica el 34\% de los casos. La probabilidad de tener un nuevo episodio de dolor de espalda vendría dada por la siguiente fórmula:

$$
\begin{aligned}
& \mathrm{P}(\text { Episodio de dolor }=1)= \\
& \frac{1}{1+\exp (-4,198-0,253 \times N D I-0,95 \times C S F)}
\end{aligned}
$$

De acuerdo con esta ecuación, un trabajador con altos valores de discapacidad cervical y bajos valores de calidad de vida (física) tienen más probabilidades de sufrir un episodio de dolor de espalda. Influyendo más estos factores en la probabilidad de dolor de espalda que la actividad física o la postura corporal (Tabla 5).

Siendo el parámetro estimado (B), su error estándar (ET) y su significación estadística con la prueba de Wald, que es un estadístico que sigue una ley de Chi Cuadrado con 1 grado de libertad y la estimación de la Odds Ratio (Exp (B)) con el intervalo de confianza para el 95\%.

\section{Discusión}

En el presente estudio la mayoría de participantes refirieron dolor de espalda en el último año, que dificultó las actividades de su vida diaria. Esta prevalencia coincide con estudios precedentes como el de Berolo et al. ${ }^{24}$, con un $52 \%$ de dolor de hombro derecho y un $62 \%$ de dolor en la parte superior de la espalda y el de Cagnie et al. ${ }^{25}$ con una prevalencia de dolor de cuello en los trabajadores de oficina del $45,5 \%$. 
Tabla 5. Regresión logística episodio de dolor.

\begin{tabular}{|c|c|c|c|c|c|c|c|c|}
\hline & \multirow{2}{*}{ B } & \multirow{2}{*}{ E.T. } & \multirow{2}{*}{ Wald } & \multirow{2}{*}{ gl } & \multirow{2}{*}{ Sig. } & \multirow{2}{*}{$\operatorname{Exp}(B)$} & \multicolumn{2}{|c|}{ I.C. $95 \%$ para $\operatorname{EXP}(B)$} \\
\hline & & & & & & & Inferior & Superior \\
\hline CSF & $-0,095$ & 0,044 & 4,697 & 1 & 0,030 & 0,910 & 0,835 & 0,991 \\
\hline NDI & 0,253 & 0,094 & 7,176 & 1 & 0,007 & 1,288 & 1,070 & 1,549 \\
\hline Constante & 4,198 & 2,314 & 3,291 & 1 & 0,070 & 66,573 & & \\
\hline
\end{tabular}

Fuente: Desarrollada por los autores.

Para el individuo el dolor de cuello-hombro se puede manifestar como episodios leves y de corta duración con una actividad limitada, como episodios recurrentes con disminución de la capacidad de trabajo y rendimiento, llegando a episodios graves e incapacitantes o discapacidad crónica. Paralelamente, para la sociedad, las consecuencias económicas muestran un patrón similar que abarca desde la baja por enfermedad a corto plazo, los períodos recurrentes de baja por enfermedad a largo plazo, hasta una posible jubilación anticipada y/o invalidez 3 .

En el caso de los trabajadores con PVD de nuestra muestra, la mayoría no tiene discapacidad cervical o bien tiene un grado de discapacidad leve, ninguno obtuvo grados de discapacidad severa o incapacidad completa. Estos datos son similares a otros estudios como el de Kim y $\mathrm{Kim}^{26}$, con una media de 4,9 y desviación típica de 3,9 en el índice de discapacidad cervical, siendo estos valores de "sin discapacidad" y "discapacidad leve”. La muestra utilizada en este estudio era sensiblemente más joven que en nuestro trabajo (estudiantes universitarios de 19 a 24 años de edad).

En cuanto a la comparación entre sexos, los resultados obtenidos indicaron que no existen diferencias para el dolor y la discapacidad cervical. Sin embargo, otros autores afirman que el sexo puede ser un factor determinante ya que la prevalencia de dolor en la extremidad superior es mayor en las mujeres que en los hombres ${ }^{25,27}$. En esta línea Cagnie et al. ${ }^{25}$, mediante un análisis multivariante reveló que las mujeres tienen un riesgo casi dos veces mayor en comparación con los hombres de tener dolor de cuello. Afirmación corroborada por Cote et al..$^{28}$ y Jensen ${ }^{29}$ que señalan también una mayor frecuencia y dolor persistente por parte de las mujeres, incluso en la adolescencia ${ }^{30}$. Entre los factores expuestos como predictores de esta diferencia se indicaba la tipología de trabajo que desempeña habitualmente el sexo femenino que implica tareas con carga estática en los músculos del cuello, repetitivos, con bajo control y altas exigencias mentales, siendo estos, posibles factores de riesgo para el dolor de cuello y hombro ${ }^{3,31}$. Esta puede ser la posible causa de nuestros resultados, dado que en la muestra, ambos sexos realizaban una ocupación laboral similar y, por tanto, el dolor y discapacidad cervical se mostró homogénea.

No obstante, no deberíamos obviar que el desequilibrio de género que aun persiste en nuestra sociedad a nivel laboral y doméstico hace que las mujeres sean el sexo más vulnerable a la sintomatología musculoesquelética. Las mujeres suelen estar segregadas a trabajos más sedentarios, repetitivos y rutinarios, además de tener menor tiempo para el ocio debido a una sobrecarga en el trabajo doméstico. Estas circunstancias hacen que las mujeres trabajadoras suelan presentar probabilidades entre 2 y 5 veces mayores que sus compañeros hombres de mostrar este tipo de cuadros de dolor y discapacidad ${ }^{27}$.

Es un hecho que los trastornos cervicales son una fuente importante de dolor que limita la actividad de los trabajadores. Lamentablemente, dada la multifactorialidad del problema, el trabajo preventivo se antoja una tarea compleja que no permite que existan estrategias genéricas que demuestren reducir la incidencia del dolor de cuello en la población trabajadora ${ }^{32}$. Esta multifactorialidad complica a su vez la comparación entre los diferentes estudios que tratan sobre esta temática.

A pesar de ello, el ejercicio físico y terapéutico, ha sido propuesto como una forma de mejorar la postura corporal y el dolor ${ }^{33}$. Moreira-Silva et al. ${ }^{34}$, revisaron la efectividad de las intervenciones de actividad física en el lugar de trabajo para reducir el dolor musculoesquelético entre los empleados, evaluando el tamaño del efecto de estos programas mediante un metaanálisis. Estos autores encontraron evidencia de que las intervenciones de actividad física en el lugar de trabajo reducen significativamente el dolor musculoesquelético general y el dolor cervical y de hombros. Por tanto, parece que los esfuerzos encaminados a crear un contexto laboral que priorice unos bue- 
nos hábitos de higiene físico y que luche contra el sedentarismo, podría ser una buena estrategia para reducir la incidencia de este tipo de cuadros de dolor en la población trabajadora.

En definitiva, el presente trabajo muestra que las variables que mejor predicen la probabilidad de que suceda un nuevo episodio de dolor de espalda es el componente físico de la calidad de vida y la puntuación del NDI, es decir, la discapacidad cervical. Obteniendo un modelo que puede considerarse como aceptable, tanto por su especificidad como sensibilidad. De esta manera, un trabajador con altos valores de discapacidad cervical y bajos valores de calidad de vida (física) tiene más probabilidad de sufrir un episodio de dolor de espalda. De ahí que, la intensidad del dolor y el ángulo cervical influyen en la probabilidad de episodio de dolor de espalda, pero no de manera significativa en nuestro modelo.

\section{Colaboradores}

T García-Remeseiro y A Gutiérrez-Sánchez trabajaron en la concepción, análisis e interpretación de los datos y en la redacción final. D Alonso-Fernández y R Graganta llevaron a cabo la interpretación de los datos y su revisión crítica. Todos los autores contribuyeron de manera fundamental para la realización del estudio y aprobaron la versión final del contenido del manuscrito.

\section{Agradecimientos}

Los autores desean dar las gracias a todos los participantes y a la Consellería de Sanidade de la Xunta de Galicia, cuya colaboración y dedicación hicieron posible este estudio.
A efectos prácticos, la pontencialidad de la presente investigación radica en la detección de una creciente sintomatología cervical derivada del uso continuo de pantallas por los individuos en su vida profesional y personal. Dicha circunstancia pone de manifiesto la necesidad de efectuar un esfuerzo de diagnóstico de esta realidad con el objetivo de establecer protocolos de prevención y rehabilitación de las patologías asociadas a este tipo de conductas.

En esta dirección, el lograr establecer un patrón de dolor en la población que trabaja diariamente con PVD y la identificación de los factores responsables de desencadenar un nuevo episodio podrían ser variables relevantes para el diseño y desarrollo de programas de prevención adaptados que intenten minimizar los efectos nocivos del uso de pantallas en la salud de los trabajadores.

\section{Referencias}

1. GBD 2015 Disease and Injury Incidence and Prevalence Collaborators. Disease and Injury Incidence and Prevalence Collaborators Global, regional, and national incidence, prevalence, and years lived with disability for 310 diseases and injuries, 1990-2015: a systematic analysis for the Global Burden of Disease Study 2015. Lancet 2016; 388(10053):1545-1602.

2. Gerr F, Marcus M, Ensor C, Kleinbaum D, Cohen S, Edwards A, Gentry E, Ortiz DJ, Monteilh C. A prospective study desing and incidence of musculoskeletal symtoms and disorders. Am J Ind Med 2002; 41:221235.

3. Larsson B, Søgaard K, Rosendal L. Work related neckshoulder pain: a review on magnitude, risk factors, biochemical characteristics, clinical picture and preventive interventions. Best Pract Res Clin Rheumatol 2007; 21:447-463.

4. Simões MRL, Assunção AÁ, Medeiros AM. Dor musculoesquelética em motoristas e cobradores de ônibus da Região Metropolitana de Belo Horizonte, Brasil. Cien Saude Colet 2018; 23(5):1363-1374.

5. Falcao IR, Couto MC, Lima VMC, Pena PGL, Andrade LL, Müller JDS, Alves IB, Viana WDS, Rego RCF. Prevalência dos distúrbios musculoesqueléticos nos membros superiores e pescoço em pescadoras artesanais/marisqueiras em saubara, Bahia, Brasil. Cien Saude Colet 2015; 20(8):2469-2480. 
6. Palacios-Cena D, Alonso-Blanco C, Hernández-Barrera, Carrasco-Garrido P, Jiménez-García R, Fernández-de-las-Peñas C. Prevalence of neck and low back pain in community-dwelling adults in Spain: an updated population-based national study (2009/102011/12). Eur Spine J 2015; 24:482.

7. Punnett L, Wegman, DH. Work-related musculoskeletal disorders: the epidemiologic evidence and the debate. J Electromyogr Kinesiol 2004; 14:13-23.

8. Juul-Kristensen B, Kadefors R, Hansen K, Byström P, Sandsjö L, Sjøgaard G. Clinical signs and physical function in neck and upper extremities among elderly female computer users: the NEW study. Eur J Appl Physiol 2006; 96:136-145.

9. McLean SM, May S, Klaber-Moffett J, Sharp DM, Gardiner E. Risk factors for the onset of non-specific neck pain: a systematic review. J Epidemiol Community Health 2010; 64:565-572.

10. Huskisson EC. Measurement of pain. Lancet 1974; 304:1127-1131.

11. Llorca GJ, Muriel C, González-Tablas MM, Díez MA. Relación entre características del dolor crónico y los niveles de depresión. Rev Soc Esp Dolor 2007; 14:2635.

12. Price DD, McGrath PA, Rafii A, Buckingham B. The validation of visual analogue scales as ratio scale measures for chronic and experimental pain. Pain 1983; 17:45-56.

13. Yarnitsky D, Sprecher E, Zaslansky R, Hemli JA. Multiple session experimental pain measurement. Pain 1996; 67:327-333.

14. Vernon H, Mior S. The Neck Disability Index: a study of reliability and validity. J Manipulative Physiol Ther 1991; 14:409-415.

15. Vernon H. The Neck Disability Index: state-of-the -art, 1991-2008. J Manipulative Physiol Ther 2008; 31:491-502.

16. Ware Jr J, Kosinski M, Keller SD. A 12-Item ShortForm Health Survey: construction of scales and preliminary tests of reliability and validity. Med Care 1996; 34:220-233.

17. Ware JE, Sherbourne CD. The MOS 36-item shortform health survey (SF-36): I. Conceptual framework and item selection. Med Care 1992; 30(6):473-483.

18. Vilagut G, Valderas JM, Ferrer M, Garin O, LópezGarcía E, Alonso J. Interpretación de los cuestionarios de salud SF-36 y SF-12 en España: componente físico y mental. Med Clin 2008; 130:726-735.

19. Vera-Villarroel P, Silva J, Celis-Atenas K, Paves P. Evaluación del cuestionario SF-12: verificación de la utilidad de la escala salud mental. Rev Med Chile 2014; 142:1275-1283.

20. García-Remeseiro T, Gutiérrez-Sánchez A, AlonsoFernández, D. Fiabilidad inter e intra-observador del software de evaluación postural (PAS/SAPO): una revisión sistemática. Rev Andaluza Med Deporte 2019; 12(3):286-290.

21. Ferreira EAG, Duarte M, Maldonado EP, Burke TN, Marques AP. Postural assessment software (PAS/ SAPO): Validation and reliabiliy. Clinics 2010; 65:675681.

22. García-Remeseiro, T, Gutiérrez-Sánchez Á. Analysis of relationships between the use of visual display terminals, craniocervical angle and physical activity: A pilot study. J Hum Sport Exerc 2017; 12:325-332.
23. Wahlund K, List T, Dworkin, SF. Temporomandibular disorders in children and adolescents: reliability of a questionnaire, clinical examination, and diagnosis. $J$ Orofac Pain 1998; 12:42-51.

24. Berolo S, Wells RP, Amick BC. Musculoskeletal symptoms among mobile hand-held device users and their relationship to device use: a preliminary study in a Canadian university population. Appl Ergon 2011; 42:371-378.

25. Cagnie B, Danneels L, Van Tiggelen D, De Loose V, Cambier D. Individual and work related risk factors for neck pain among office workers: a cross sectional study. Eur Spine J 2007; 16:679-686.

26. Kim EK, Kim JS. Correlation between rounded shoulder posture, neck disability indices, and degree of forward head posture. J Phys Ther Sci 2016; 28:29292932.

27. Strazdins L, Bammer G. Women, work and musculoskeletal health. Soc Sci Med 2004; 58:997-1005.

28. Cote P, Cassidy JP, Carroll LJ, Kristman V. The annual incidence and course of neck, and shoulder pain in the general population: a population based cohort study. Pain 2004; 112:267-273.

29. Jensen C. Development of neck and hand-wrist symptoms in relation to duration of computer use at work. Scan J Work Environ Health 2003; 29:197-205.

30. Piola TS, Bacil EDA, Pacífico AB, Camargo EM, Campos W. Nível insuficiente de atividade física e elevado tempo de tela em adolescentes: Impacto de fatores associados. Cien Saude Colet 2020; 25(7):2803-2812.

31. Treaster DE, Burr D. Gender differences in prevalence of upper extremity musculoskeletal disorders. Ergonomics 2004; 47:495-526.

32. Côté P, Van der Velde G, Cassidy JD, Carroll LJ, Hogg-Johnson S, Holm LW, Carragee EG, Haldeman S, Nordin M, Hurwitz EL, Guzman J, Peloso PM. The burden and determinants of neck pain in workers. Eur Spine J 2008; 17:60-74.

33. García-Remeseiro T. Programa de higiene postural y actividad física en el ámbito laboral con trabajadores de pantallas de visualización de datos (PVD). En: Diseño y evaluación de programas educativos en el ámbito social. Actividad fisica y dramateria. Madrid: Alianza Editorial; 2017. p. 251-275.

34. Moreira-Silva I, Teixeira PM, Santos S, Abreu S, Moreira C, Mota J. The effects of workplace physical activity programs on musculoskeletal pain: A systematic review and meta-analysis. Workplace Health Saf 2016; 64:210-222.

Artículo presentado en 29/10/2018

Aprobado en 08/10/2019

Versión final presentada en 10/10/2019

Editores jefes: Romeu Gomes, Antônio Augusto Moura da Silva 